\title{
Potencialidades e impactos ambientais dos resíduos oriundos da malacocultura
}

\section{Potential and environmental impacts of waste from the malacoculture}

\author{
Marcos Eduardo Miranda Santos* \& Efigenia Magda de Oliveira Moura \\ Departamento de Química e Biologia, Universidade Estadual do Maranhão - Uema
}

*E-mail: markoseduardo2008@hotmail.com

Recebido: 30 de agosto de 2017 / Aceito: 21 de setembro de 2017 / Publicado: 17 de dezembro de 2017

\begin{abstract}
Resumo O cultivo de moluscos bivalves é conhecido como malacocultura. Esta atividade é considerada pela Organização de Agricultura e Alimento das Nações Unidas (FAO/ONU) como uma atividade ecologicamente correta, pois promove a preservação e manutenção dos recursos naturais marinhos. No entanto, densidades elevadas de cultivo de moluscos podem causar determinados impactos ambientais provocando desequilíbrios nos ecossistemas, devido a uma maior produção dos resíduos provenientes da malacocultura. Desta forma, buscou-se, através de pesquisa bibliográfica, apresentar os impactos ambientais causados pela deposição inapropriada dos resíduos gerados na malacocultura descritos na literatura cientifica, bem como, sugerir possíveis formas de utilização destes resíduos de modo a promover o desenvolvimento sustentável e responsável dessa atividade com minimização dos impactos ambientais por ela gerados. Dentre os principais impactos estruturais citados na literatura estão o assoreamento e a eutrofização do ambiente marinho, os quais representam sérios riscos à saúde humana, sendo necessária a realização de ações educativas a fim de reduzir os impactos ambientais causados por esses resíduos, e evitar o desperdício de grande quantidade de matéria-prima.
\end{abstract}

Palavras-chave: moluscos bivalves, impactos ambientais, resíduos.

\begin{abstract}
The cultivation of bivalve molluses is known as malacoculture. This activity is considered by the Food and Agriculture Organization of the United Nations (FAO / ONU) as an ecologically correct activity, as it promotes the preservation and maintenance of marine natural resources. However, high densities of mollusc cultivation can cause certain environmental impacts causing ecosystem unbalances due to increased production of the malacoculture residues. In this way, it was sought, through bibliographical research, to present the environmental impacts caused by the inappropriate deposition of waste generated in the malacoculture described in the scientific literature, as well as suggest possible ways of using these residues in order to promote the sustainable and responsible development of this activity with minimization of the environmental impacts generated by it. From the main structural impacts cited in the literature, there are silting and eutrophication of the marine environment, which represent serious risks to human health, and becames necessary to carry out educational actions to reduce the environmental impacts caused by these residues, and avoid waste of large amount of raw material.
\end{abstract}

Key words: bivalve molluscs, environmental impacts, residues. 


\section{Introdução}

A Classe Bivalvia (Filo Mollusca) reúne animais popularmente conhecidos por ostras, mexilhões, sururus, sarnambis, berbigões, vieira, entre outros. Estes animais possuem um corpo comprimido lateralmente, protegido por uma concha calcária composta por duas valvas dorsalmente articuladas. São animais filtradores que podem ser encontrados tanto em ambientes marinhos, como em ambientes dulcícolas (Brusca; Brusca, 2002). Muitas espécies de moluscos bivalves são de interesse econômico, sendo utilizadas principalmente na produção de pérolas e na alimentação. Quando comparados a outros tipos de pescado, os moluscos apresentam em sua carne um alto teor de carboidratos e menores concentrações de nitrogênio (Silva 2007).

O cultivo de moluscos bivalves é conhecido como malacocultura. O Brasil é o segundo maior produtor de moluscos bivalves da América Latina (Brasil, 2015), resultado do desenvolvimento da atividade de aquicultura, que passou por avanços consideráveis nos últimos 10 anos. No Brasil esta atividade representa a maior parcela da produção mariculturista (Brasil, 2015). Os maiores produtores brasileiros de moluscos bivalves são os estados de São Paulo, Rio de Janeiro, Espírito Santo e Santa Catarina, sendo que o último vem liderando a atividade malacoculturista nos últimos anos devido a sua geografia favorável e a qualidade da água da região (Custódio, 2005).

A malacocultura é considerada pela Organização de Agricultura e Alimento das Nações Unidas (FAO/ONU) uma atividade ecologicamente correta, pois promove a preservação e a manutenção dos recursos naturais marinhos (Barbieri et al., 2014; Suplicy, 2005). Esta atividade é reconhecida mundialmente como uma importante alternativa de geração de empregos, renda e alimento, que tem contribuído para a fixação de comunidades tradicionais em seus locais de origem (Barbieri et al., 2014).

No entanto, as densidades elevadas de moluscos bivalves produzidas pelo cultivo podem causar impactos ambientais provocando desequilíbrios nos ecossistemas, devido a uma maior produção dos resíduos sólidos. Em muitas mariculturas, conchas de moluscos têm sido descartadas em locais inadequados, ocasionando poluição ambiental e visual, e quando dispostas nas praias, desconforto aos banhistas e prejuízos à paisagem (Paola et al., 2012; Filho et al., 2014).

As conchas representam o peso majoritário dos bivalves e esse material está sendo depositado de forma incorreta, com a maior parte sendo devolvida ao mar ou depositados nas praias, aterros ou lixões, mas não há destino apropriado ou reutilização. Com esse sistema de deposição, uma quantidade razoável de recursos naturais está sendo desprezada. O Brasil, portanto, carece de trabalhos específicos voltados ao aproveitamento desses recursos (Rosa, 1997; Silva, 2007).

Dessa forma, buscou-se com este trabalho, apresentar os principais impactos ambientais causados pela deposição inapropriada dos resíduos da malacocultura, descritos na literatura cientifica, bem como sugerir possíveis formas de utilização destes resíduos de modo a promover o desenvolvimento sustentável e responsável dessa atividade por meio da minimização dos impactos ambientais por ela gerados.

\section{Material e Métodos}

A compilação dos dados foi realizada através de revisão na literatura com bases na literatura cinza (resumos publicados em congressos, monografias, dissertações e teses), livros e artigos científicos, seguindo recomendações de Leal et al. (2013). Para busca de tais trabalhos se fez uso de ferramentas disponíveis na web, como Google Acadêmico (http://scholar.google.com.br), Scientific Electronic Library Online - Scielo (www.scielo.org/php/index.php) e Web of Science - WoS (http://portal.isiknowledge.com) (Miretzki, 2003). A busca foi realizada com a combinação de palavras-chave como "moluscos", "bivalves", "conchas", "resíduos" e "impactos ambientais".

\section{Resultados e Discussão}

\section{IMPACTOS DA DEPOSIÇÃO INADEQUADA DE RESÍDUOS DA MALACOCULTURA}

A priori, é importante destacar que os resíduos produzidos pelos moluscos bivalves são benéficos ao ambiente marinho; o problema se encontra na produção intensiva destes resíduos, como ocorre em alguns cultivos de moluscos, que gera uma quantidade muito maior de conchas e outros resíduos, quando comparada com a produção natural, causando assim impactos ao ambiente.

Conforme afirma Bocchese (2008), os resíduos gerados no cultivo de moluscos bivalves, em especial as conchas calcárias de ostras e mexilhões representam um grande problema ambiental quando do seu descarte 
inapropriado devido aos inúmeros impactos que causam: odores em terrenos de acumulação do material descartado, poluição visual, assoreamento de áreas de cultivo, danos à atividade turística, alterações locais nos padrões físico-químicos da água, entre outros.

Quando descartados em terrenos baldios, estes resíduos acabam também por atrair roedores e insetos que se alimentam de matéria orgânica, colocando em perigo a saúde da população que vive próxima ao local onde está sendo descartado o resíduo, uma vez que tais animais podem ser vetores de doenças (Chierighini et al, 2011).

Quando lançados ao mar, estes resíduos, por terem matéria orgânica, podem causar o fenômeno chamado "eutrofização" (do grego $e u$, bem, bom, e trofos, nutrição), que é o aumento de nutrientes disponíveis no ambiente aquático (Odum, 1983) com consequente proliferação de microrganismos, como dinoflagelados (protistas fotossintetizantes), que causam por sua vez outro fenômeno, as marés vermelhas, assim denominadas devido à coloração que esses organismos conferem a água

Outro impacto que o descarte de grandes quantidades de conchas pode causar em ambientes aquáticos refere-se ao acúmulo desse material no sedimento, o que ao longo dos anos pode causar o assoreamento do local (Chierighini et al., 2011).

Lemos et al. (2007) identificou locais na Baía de Florianópolis onde existe dificuldade de passagem das baleeiras, devido ao assoreamento provocado pelo acúmulo de resíduos ali lançados.

Além das conchas, outros resíduos sólidos gerados na malacocultura são as fezes e pseudofezes dos moluscos - porção de alimento filtrado que é rejeitado junto com o muco ingerido após uma seleção do sistema filtrador do animal em busca de partículas de maior teor orgânico (Barbieri et al., 2014). Juntas (fezes e pseudofezes) formam o que é chamado de biodepósito (Suplicy, 2005). A biodeposição, quando em grande quantidade, torna-se um problema não só de caráter ambiental, mas também econômico, visto que o acumulo de biodepósitos no sedimento altera os fluxos das correntes de água, transformando estes locais em regiões anóxicas, prejudicando assim o cultivo (Quesada et al., 1998), aumentando a demanda biológica de oxigênio e a produção de metano, ácido sulfídrico - considerado indicador de ambientes poluídos por matéria orgânica - e lipídios nas zonas marinhas (Holmer; Kristensen, 1992; Henderson et al., 1997; Vinatea; Vieira, 2005) além de ocasionarem diminuição da abundância da macrofauna bêntica sob os locais de cultivo (Jaramillo et al., 2000). O aumento da matéria orgânica sob os sistemas de cultivos de moluscos leva também ao aumento na abundância de poliquetas oportunistas, mudanças na rede trófica e diminuição da diversidade (Jambrina, 2000).

No entanto, Suplicy (2005) afirma que os biodepósitos só são um problema quando o cultivo é implantado e operado acima das densidades impostas pela capacidade sustentável do local ou em locais rasos com limitada circulação de água, pois, quando em quantidades moderadas, o que é biodepositado passa a servir de alimento para animais detritívoros, incluindo muitos vermes e crustáceos, que por nsua vez, servem de alimento para peixes e aves.

Outros resíduos da malacocultura são os efluentes líquidos gerados nos locais de cultivo que muitas vezes são jogados sem nenhum tratamento no mar, o que, tal como ocorre com as conchas pode levar a eutrofização destes ambientes. Além disso, outro problema ambiental relacionado aos efluentes, mas que não está ligado diretamente ao seu descarte, diz respeito ao uso de água doce para o cultivo, como descrito por Petrielli (2008), o que muitas vezes resulta em grandes volumes de água desperdiçados, por isso, é recomendado aos malacocultores o uso de água do mar para o cultivo, de forma a permitir a conservação da água doce.

\section{POTENCIALIDADES DOS RESÍDUOS DA MALACOCULTURA}

É importante que se busquem formas corretas de se descartar, e se possível, reutilizar os resíduos, sejam eles sólidos ou líquidos, gerados nas atividades malacoculturistas.

Existem diversas formas de reutilizar esses resíduos. No caso das conchas, por serem constituídas principalmente por carbonato de cálcio $\left(\mathrm{CaCO}_{3}\right)$, podem ser aproveitadas na produção de cal virgem, cal hidratada, carga em polímeros, blocos e pavimentos para construção civil, construções de estradas, pasta de papel (substituindo em parte a matéria-prima vegetal), mármore compacto para pavimentos e revestimentos, em adubos, pesticidas e rações; podem ser utilizadas também na indústria de cerâmica, tijolos, tintas, espumas de polietileno, na produção de talco, vidros, na indústria do cimento, na produção de vernizes e borrachas, na correção de solos (calagem), impermeabilização de lagoas, selagem de lixeiras (material impermeabilizante) e em medicamentos, principalmente naqueles de combate a osteoporose (Boicko et al., 2004; Bocchese, 2008; Chierighini et al., 2011).

Bocchese (2008) destaca que para se reutilizar os resíduos da malacocultura é necessário que se elimine a matéria orgânica e possíveis organismos que estejam aderidos à superfície das conchas. Uma forma de 
processar as conchas para obtenção do carbonato de cálcio seria a moagem a úmido, seguida de tratamento térmico $\left(400-500^{\circ} \mathrm{C}\right)$, para eliminação da água da moagem e retirada do substrato orgânico das conchas. Esta forma de processamento tem a desvantagem de ser muito onerosa, dificultando a utilização desses resíduos como matéria-prima renovável. Desta forma, a utilização das conchas descartadas dependerá da disponibilidade mensal, da logística de recolhimento e de transporte e, ainda, de baixo custo no processamento para eliminação da matéria orgânica aderida às conchas. É possível fazer uso das conchas descartadas pela malacocultura, desde que exista disponibilidade regular mensal do produto, logística eficiente de recolhimento e de transporte e custo final do produto compatível com o de mercado.

Estudos descritos na literatura confirmam a viabilidade de conchas para os usos descritos acima. Boicko et al. (2004) verificou que o pó extraído das conchas pode ser utilizado como aditivo de carga na fabricação de PVC. A adição de carbonato de cálcio resulta em produtos de boas propriedades mecânicas e com boa possibilidade de pigmentação, podendo agir como agente nucleante, aumentando a durabilidade, além de facilitar o processo de extrusão. O produto entra na composição de perfis, tubos para água e pisos, revestimentos, fios e cabos elétricos.

O carbonato de cálcio é utilizado na fabricação de tubos de PVC, pois é necessária a incorporação de aditivos para que o PVC passe de resina plástica para o produto final. Esses aditivos tem a finalidade de baixar o custo ou melhorar determinada propriedade dependendo da utilização do PVC pronto.

A indústria farmacêutica também tem se beneficiado do carbonato de cálcio extraído das conchas de moluscos. Um exemplo são os suplementos alimentares que auxiliam no combate e prevenção da osteoporose. Estudos feitos com pessoas idosas, no Japão, confirmam que o carbonato extraído das conchas é mais bem absorvido pelo intestino e aumenta a densidade mineral dos ossos (Fujita et al., 1990), além disso cientistas americanos mostraram que o cálcio de concha de ostra reduziu em 50\% os sintomas da tensão pré-menstrual, diminuindo consideravelmente a depressão e as cólicas em mulheres (Sant́Anna et al., 2007).

Outra aplicabilidade das conchas confirmada pela ciência é o uso destes resíduos como materiais de construção. Na Coréia do Sul (Ásia) foi estudada a possibilidade da substituição de agregados miúdos por conchas de ostras moídas na fabricação de argamassa, principalmente para usos mais viáveis como materiais para preenchimento na construção de diques. Esta foi considerada uma boa alternativa em casos de pouca disponibilidade de areia (Yoon et al., 2003; Yang et al, 2005; Petrielli, 2008).

Como suplemento na ração animal, percebeu-se que a farinha de ostra garante um contínuo suprimento de cálcio evitando a ocorrência de doenças ligadas à má formação óssea de aves e suínos. Garlich e Parkhurst (1982), porexemplo, perceberam que o uso de conchas de ostras em ração para poedeiras resultou em redução da taxa de declínio da produção, menor número de ovos com casca fracas e dobro de ovos comerciais produzidos de 2 a 5 dias do período de jejum.

Estudos realizados na Flórida (EUA) e Coréia do Sul (Ásia) revelam que as conchas de ostras, após serem pirolisadas a uma temperatura de $750^{\circ} \mathrm{C}$ durante 1 hora numa atmosfera de nitrogênio, transformam-se num produto com eficiência maior que $98 \%$ para remoção de fosfatos em águas residuárias, sendo esta uma importante estratégia para o controle da eutrofização de águas (Kwon et al., 2004; Petrielli, 2008).

As conchas também podem ser úteis no tratamento individual de efluentes domésticos. No Brasil, pesquisadores do Cefet/PR analisaram a eficiência de uma estação de esgoto por meio de Zona de Raízes.A alternativa utiliza conchas de ostras em camadas onde geralmente utiliza-se seixo rolado ou cascalho no sistema de zona de raízes. Segundo os autores, a presença das conchas aumentou em $56,24 \%$ a presença de cálcio no efluente. Os íons de cálcio ligam-se facilmente ao fosfato formando fosfatos de cálcio que são rapidamente lixiviados. No entanto, é necessário renovar periodicamente as conchas do tanque, devido à concentração de poluentes pela saturação de fósforo nas conchas (Presznhuk et al., 2003; Petrielli, 2008).

Silva (2007) demonstrou que quando ativadas em condições específicas, as conchas podem transformar-se em um produto efetivo para remoção de metais, como o cobre e o fosfato. Para o tratamento de lixiviados (chorume) o autor encontrou redução de $22 \%$ nas taxas da Demanda Bioquímica de Oxigênio(DBO) e ligeiro acréscimo (7\%) nos níveis da Demanda Química de Oxigênio (DQO).

Filho et al (2014) em estudo sobre adsorção de corantes têxteis demonstrou a eficiência das conchas de moluscos na adsorção do corante reativo Remazol vermelho (RR133). Este composto, presentes na composição de efluentes industriais, apresentam grande potencial para contaminação de recursos hídricos. As conchas apresentaram potencial para o tratamento de soluções do corante, visto que apresentou remoção superior a 98\%. O uso de conchas na adsorção destes corantes configura-se em uma alternativa economicamente barata e ecologicamente sustentável para o tratamento destes efluentes.

Os estudos sobre cultivos de moluscos bivalves em sua maioria referem-se à biologia da espécie, cultivo, estoques naturais, legislação e normatização, manejo, comercialização, entre outros, mas poucos têm 
contemplado os impactos e as possíveis utilizações dos resíduos gerados no cultivo desses animais (Silva, 2007). Portanto, fazem-se necessários trabalhos específicos voltados para o descarte e aproveitamento correto desses recursos, visando um desenvolvimento sustentável da atividade malacoculturista.

\section{Comentários Conclusivos}

A discussão sobre os impactos ambientais gerados pelo descarte inapropriado dos resíduos provenientes da malacocultura ainda não está consolidada na literatura cientifica brasileira e internacional, carecendo de mais trabalhos que discutam esta temática. Entre os principais impactos citados na literatura estãoo assoreamento ea eutrofização do ambiente marinho, que representam sériosriscos à saúde humana.

Existe um grande leque de possibilidades nas quais os resíduos provenientes da malacocultura podem ser utilizados, sendonecessária a realização de ações educativas com malacocultores a fim de socializar este conhecimento, reduzindo assim os impactos ambientais causados por esses resíduos e evitando o desperdício de grande quantidade de matéria-prima proporcionando desenvolvimento social com a geração de renda e por conseguinte a fixação de comunidades costeiras.

\section{Referências}

Barbieri, E.; Marquez, H.L.A.; Campolim, M.B. \& Salvarani, P.I. (2014). Avaliação dos Impactos ambientais e socioeconômicos da aquicultura na região estuarina-lagunar de Cananéia, São Paulo, Brasil. RGCI, 14 (3): 385-398,

Brasil (2015). Aquicultura. [web page]. Ministério da Pesca e Aquicultura, Brasília, Brasil. Acessado em: $<$ http://mpa.gov.br/index.php/aquicultura>.

Bocchese, D. C. F. (2008). Eliminação de matéria orgânica de conchas de ostras por processo biológico. [Monografia]. Florianopolis (SC): Universidade Federal de Santa Catarina.

Boicko, A.L.; Hotza, D. \& Sant'anna, F.S.P. (2004). Utilização de Conchas da Ostra Crassostrea Gigas como Carga para Produtos de Policloreto de Vinila (PVC). In: Simpósio Internacional de Qualidade Ambiental. Porto Alegre: Anais do Simpósio Internacional de Qualidade Ambiental, 4

Brusca, R.C. \& Brusca, G.J. (2007). Invertebradoss. $2^{\mathrm{a}}$ ed. Rio de Janeiro: Guanabara Koogan, 968 p.

Chierighini, D.; Bridi, R.; Rocha, A.A. \& Lapa, K. R. (2011). Possibilidades do uso das conchas de moluscos. III International Workshop advances in cleaner production.

Custódio, A. V. (2005). MPEs inseridas em arranjos produtivos locais: um estudo de caso da malacocultura na Grande Florianópolis/SC. [Dissertação de Mestrado]. Florianopolis (SC): Universidade Federal de Santa Catarina.

FAO (2004). Marine Biotoxins. FAO Food and Nutrition Paper, Roma, n. 80.

Filho, R.B.A.; Menezes, G.A. Ferreira, J.M. \& Sobrino, M.A.M. (2014). Avaliação do resíduo da malacocultura como adsorvente do corante Remazol vermelho RR133. Anais do X Encontro Brasileiro sobre Adsorção, Guarujá.

Fujita, T.; Fukase, M.; Miyamoto, H.; Matsumoto, T. \& Ohue, T. (1990). Increase of bone mineral density by calcium supplement with oyster shell electrolysate. Bone Miner, 11 (1): 85-91.

Garlich, J.D. \& Parkhurst, C.R. (1982). Increased egg production by calcium supplementation during the initial period of a forced molt. Poult. Sci., 61: 955-961.

Henderson, R.J.; Forrest, D.A.M.; Black, K.D. \& Park, M.T. (1997). The lipid composition of sea loch sediments underlying salmon cages. Aquaculture, 158 (1): 69-83.

Holmer, M. \& Kristensen, E. (1992). Impact of marine fish cage farming on metabolism and sulfate reduction of underlying sediments. Marine Ecology Progress Series, 80: 191-201.

Jambrina, L. M. C. (2000). Aproximação metodológica ao diagnóstico de áreas litorâneas com aptidão para maricultura: aplicações no Estado de São Paulo. [Tese de Doutorado]. São Paulo (SP): Universidade de São Paulo.

Jaramillo, E.; Beltrán, C. \& Bravo, A. (1992). Musselbiodeposition in estuary in southern Chile. Marine Ecology Progress Series, 82: 85-94.

Kwon, H.; Lee, C.; Jun, B., Yun, J.; Weon, S. \& Koopman, B. (2004). Recycling waste oyster shells for eutrophication control. Resources, Conservation \& Recycling, 41: 75-82.

Lemos, S.S.; Sant'anna, F.S.P; Silva, F.A; Santos, C.L. \& De Cesario, F. (2007). Avaliação socioambiental do destino e da reutilização dos resíduos da ostreicultura. SEPEX.

Odum, E. P. (1983). Ecologia. Rio de Janeiro: Guanabara Koogan.. 
Paola, A. V. M.; Antonio, T. M.; Valdeir E. J. \& Ivan, C.A.R.D.T. (2012). Utilization of ground clam shells in the adsorption of phosphorus and for correction of soil acidity. Eng. Agríc., 32 (5): 866-874.

Pereira, A.; Teixeira, A.L.; Poli, C.R.; Brognoli, F.F.; Silva, F.C.da; Rupp, G.S.; Silveira J. R., N.; \& Araújo, S.C. (1998). Biologia e Cultivo de Ostras. UFSC.

Petrielli, F. A. S. (2008). Viabilidade técnica e econômica da utilização comercial das conchas de ostras descartadas na localidade de Ribeirão da Ilha, Florianópolis, Santa Catarina. [Dissertação de Mestrado], Florianópolis (SC): Universidade Federal de Santa Catarina.

Presznhuk, R.A.O.; Van Kaick, T.S.; Casagrande Jr, E.F. \& Umezawa, H.A. (2003). Tecnologia apropriada e saneamento: análise de eficiência de estações de tratamento de esgoto por meio de zona de raízes, Atas da Semana de Tecnologia: Tecnologia para quem e para quê? Um olhar interdisciplinar. Curitiba: Editora Cefet-PR.

Quesada, J.E. \& Coelho, M.A.; Aquini, E.N. (1998). Aquicultura sustentável: construindo um conceito. In: Aquicultura Brasil. Recife: Anais do II Aquicultura Brasil, 2.

Rosa, R. de C. C. (1997) Impacto do cultivo de mexilhões nas comunidades pesqueiras de Santa Catarina. Florianópolis. [Dissertação de Mestrado], Florianopólis (SC): Universidade Federal de Santa Catarina.

Sant'Anna, F. S. P. et. al. (2007). Projeto Valorização dos Resíduos da Maricultura. SubProjeto 3: Soluções Tecnológicas Para o Aproveitamento de Conchas de Ostras. [Dissertação de Mestrado], Florianopólis (SC): Universidade Federal de Santa Catarina.

Silva, D. (2007). Resíduo sólido da malacocultura: caracterização e potencialidade de utilização de conchas de ostras (Crassostrea gigas) e mexilhão (Perna perna). [Dissertação de Mestrado], Florianopólis (SC): Universidade Federal de Santa Catarina.

Suplicy, F. M. (2005). Cultivo de moluscos: Uma atividade que produz inúmeros impactos ambientais positivos. Panorama da Aquicultura, 9 (5): 27-31.

Vinatea, L. \& Vieira, P. Modos de apropriação e gestão patrimonial de recursos costeiros: o caso do cultivo de moluscos na baía de Florianópolis, Santa Catarina. B. Inst. Pesca, 31 (2): 147 - 154.

Yang, E.; Yi, S. \& Leem, Y. (2005). Effect of oyster shell substituted for fine aggregate on concrete characteristics: Part I. Fundamental properties. Cement and Concrete Research. 35 (11): 2175-2182.

Yoon, G.; Kim, B.; Kim, B. \& Han, S. (2003). Chemical-mechanical caracteristical of crushed oyster-shell. Waste Management, 23: 825-834. 